\title{
La protection de l'homme contre les rayonnements ionisants et contre les autres agents nocifs
}

\author{
H. JAMMET $\left(^{\star}\right)$ et M. DOUSSET $\left({ }^{\star \star}\right)$
}

(Manuscrit reçu le 12 novembre 1981)

\begin{abstract}
RÉSUMÉ
Après avoir comparé d'une façon générale l'état de nos connaissances sur les effets pathologiques des rayonnements ionisants et sur ceux des différentes substances toxiques qui peuvent être présentes dans l'air, ce travail s'attache à confronter les politiques de protection mises en œuvre tant du point de vue de la cohérence des doctrines que de leur degré d'universalité et d'acceptation dans le monde.
\end{abstract}

\section{ABSTRACT}

Our knowledge on the pathological effects of ionizing radiation as well as of various airbone toxicants is first reviewed. The protection policies implemented are compared both from the viewpoint of doc. trine consistency and degree of universality and world-wide acceptance.

Entreprendre une étude comparative générale des caractères qui différencient, à l'échelle de l'ensemble des pays développés, la protection de l'homme contre cette nuisance particulière que sont les rayonnements ionisants et la protection de l'homme contre les autres nuisances auxquelles il est exposé aussi bien dans ses activités professionnelles que dans sa vie de tous les jours, serait une tâche considérable et sûrement très difficile à mener à bien. II n'est donc pas question de se livrer ici à une telle étude.

On se limitera simplement à rappeler les caractères essentiels de la protection radiologique et à situer, en se référant aux mêmes critères, l'état de la protection de l'homme pour une seule catégorie de nuisances non radiologiques, choisie pour des raisons pratiques de disponibilité de documentation: les substances toxiques dans l'air.

La santé de l'homme peut être définie d'une façon positive comme "l'état de complet bien-être physique, mental et social" [1] et tout phénomène physique, moral ou social qui peut altérer cet état de complet bienêtre peut être appelé "nuisance".

(*) Commissariat à l'énergie atomique, IPSN, Département de protection, BP 6 . 92260 Fontenay-aux-Roses.

(**) 7, rue de la Gruerie, 91190 Gif-sur-Yvette. 
Ici, cependant, ce mot sera employé dans un sens plus restreint et plus technique, entendant par là tout phénomène physique ou tout agent chimique ou biologique incorporé qui peut entraîner une altération de la santé. Un jugement moral élémentaire conduit à l'obligation de protéger l'homme contre ces agents nuisibles qui très souvent d'ailleurs sont produits par ses propres activités.

Si l'on donne au mot "politique" son sens le plus large et le plus noble, on peut dire que les formes que revêtent les diverses façons de protéger l'homme des différents types de nuisances sont des "politiques" qui débouchent sur des impératifs réglementaires édictés par les pouvoirs publics. Mais ces décisions politiques ne peuvent être prises d'une façon rationnelle qu'en s'appuyant sur l'état des connaissances obtenues par les sciences appliquées qui étudient les effets pathologiques de ces nuisances.

Dès lors, pour juger de la qualité d'une politique de la protection de l'homme contre un agent nocif donné, il est logique de s'attacher à l'examen de deux critères généraux:

- l'état d'avancement des connaissances scientifiques théoriques et expérimentales sur lesquelles elle se fonde ;

- la cohérence et l'unité synthétique plus ou moins parfaites de la doctrine ainsi que le degré d'universalité dans l'acceptation de cette doctrine.

\section{LES CONNAISSANCES}

L'ensemble des informations sur lesquelles se fonde notre connaissance des effets pathologiques des rayonnements ionisants et des substances radioactives est considérable.

Dès la découverte des rayons X (1895) par Roentgen, leur action nocive sur les tissus était constatée et des études expérimentales étaient aussitôt entreprises sur l'atteinte de la peau, du système nerveux, des ovaires, des yeux, etc. Après la découverte de la radioactivité naturelle, on ne tarda pas à constater la même action nocive des rayonnements émis par les éléments de la chaîne de l'uranium et particulièrement par le radium. Dès 1902, on observera l'apparition de cancers sur des radiodermites et la démonstration expérimentale de l'effet carcinogène des rayonnements fut apportée en 1910 .

Depuis lors, la découverte de la radioactivité artificielle en 1934 par I. et $F$. Joliot-Curie, la mise en service du premier réacteur nucléaire en 1942 à Chicago ont marqué le début d'un âge où les rayonnements et les corps radioactifs sont devenus des sources d'énergie et des instruments de travail de plus en plus utilisés en même temps que le danger potentiel important qu'ils constituaient stimulait les recherches tant expérimentales que théoriques sur leur action pathologique.

Mais c'est surtout après la guerre de 1939-1945, à la suite du choc psychologique considérable que furent, pour le monde, les explosions nucléaires d'Hiroshima et de Nagasaki, que se développèrent en même temps que les différentes applications pacifiques et militaires de l'énergie nucléaire les études systématiques sur les différentes modalités d'action biologique 
des rayonnements. On découvrit leur action mutagène, on étudia les survivants d'Hiroshima et de Nagasaki, les malades traités par radiothérapie, les travailleurs impliqués dans des accidents d'irradiation ou ayant travaillé dans des conditions jugées aujourd'hui peu satisfaisantes; de nombreux laboratoires se créèrent dans tous les pays développés et entreprirent des recherches expérimentales dont les résultats étaient aussitôt publiés dans de nombreuses revues internationales.

Pour avoir une idée de la somme considérable d'informations obtenues au cours de ces vingt-cinq dernières années, il suffit de compulser les références bibliographiques des très importants documents que sont les rapports à l'assemblée générale du Comité scientifique des Nations Unies sur les effets des rayonnements ionisants [2]. Ces rapports (le premier a été publié en 1958, le plus récent en 1977 et le $8^{\mathrm{e}}$ est en préparation) collectent, classent et analysent toutes les connaissances acquises à la date de leur publication. Ils constituent un instrument de travail unique pour les chercheurs. D'emblée, toutes ces connaissances sont confrontées et intégrées à un niveau international. On insistera plus loin sur le rôle éminent joué par la Commission internationale de protection radiologique (CIPR) pour unifier ce savoir et en tirer les conséquences doctrinales et pratiques pour la protection de l'homme.

Le niveau de connaissance ainsi atteint permet de prendre en compte tous les effets : effets aigus et effets chroniques, effets précoces et effets tardifs, effets aléatoires et effets non aléatoires, effets somatiques sur l'embryon, le fœtus, l'enfant et l'adulte et les effets éventuels sur les générations futures. Certes, il demeure encore de nombreuses incertitudes et des domaines importants restent à explorer, mais le niveau atteint est très élevé et les lignes essentielles de la connaissance des phénomènes sont acquises. Ceci permet d'établir des bilans sérieux qui pourront conduire à des décisions prudentes et équilibrées.

Face à cet ensemble cohérent de connaissances sur les rayonnements ionisants, un premier regard sur l'état du savoir relatif aux substances et autres agents toxiques donne l'impression d'une dispersion considérable. Celle-ci est due pour une grande part à l'extrême diversité des modalités d'action des différents toxiques qu'il s'agisse de ceux que l'on classe dans la catégorie des substances inorganiques, dans celle des substances organiques ou dans celle des aérosols solides ou liquides ... On a le sentiment que chaque cas est un cas particulier et qu'il y a pour chaque toxique une science propre présentant une certaine autcnomie.

Depuis le début de l'ère industrielle, l'action nocive de nombreux agents toxiques est connue et un très grand nombre d'études tant expérimentales qu'épidémiologiques ont été menées au fur et à mesure que la toxicité de ces agents a été découverte. Les références figurant dans les grands traités de toxicologie permettent de se faire une idée de la somme considérable des travaux effectués sur les effets de toutes ces substances sur l'organisme. Si l'on était capable d'en faire le décompte et la synthèse, peut-être constaterait-on qu'ils sont plus importants que ceux qui ont été consacrés à l'étude des effets des rayonnements ionisants. Mais ici cette synthèse manque; malgré les efforts de l'OMS, l'analogue du rapport du Comité scientifique des Nations Unies n'existe pas pour les toxiques non radioactifs. Cela est très certainement dû au fait qu'il n'y a pas eu, il y a 
vingt ans, dans ce domaine, une situation analogue à celle qu'ont créée, pour la radioactivité, les essais nucléaires aériens effectués par les grandes puissances entre 1953 et 1962 . La crainte que fit naître, pour l'humanité, l'augmentation générale de la radioactivité joua un rôle déterminant, aussi bien au niveau des organismes internationaux que des pouvoirs publics des différentes nations, pour que soit créé le Comité scientifique des Nations Unies et qu'un effort général soit fait par tous les pays développés pour intensifier les recherches.

Cependant, on peut se demander si l'existence d'un tel organisme de synthèse eût suffi à faire apparaître une unité cohérente du savoir permettant d'établir, comme pour les rayonnements ionisants, une base simple et universelle d'évaluation des risques pour la santé ; car, finalement, les problèmes posés par les rayonnements ionisants et les substances radioactives sont simplifiés si on les compare à la complexité de ceux que pose l'action des autres agents toxiques. On pourrait dire que pour chacun de ces derniers les risques sont spécifiques qu'il s'agisse:

- des risques de toxicité aiguë avec des atteintes pouvant se manifester au niveau des muqueuses (yeux, système respiratoire,...), du système cardio-vasculaire, des fonctions endocrines, des fonctions du système nerveux central, etc. ;

- des risques de toxicité à relativement long terme par effet cumulatif comme celui des insecticides organo-halogénés dans les organes riches en lipides ou celui des dérivés fluorés, de l'arsenic, des métaux lourds, etc. qui forment des combinaisons stables avec certains constituants de l'organisme ;

- des risques se manifestant à très long terme d'induction d'affections malignes ou d'effets génétiques. Comme on le soulignera plus loin, c'est pour les substances présentant ce dernier caractère cancérigène ou mutagène que l'on peut espérer parvenir dans des délais raisonnables à une certaine unification du savoir $[3,4]$.

Finalement, on peut dire que, si l'on se fixe comme idéal en vue des applications pratiques un savoir cohérent permettant de déboucher sur une politique simple de la protection, les connaissances en matière de nuisances non radioactives présentent un certain retard par rapport à nos connaissances sur les rayonnements ionisants. Pour la plus grande part, ce retard est dû à la complexité des phénomènes mis en jeu et, peut-être, après tout, est-ce un leurre que de vouloir trouver simplicité et cohérence dans un domaine où la matière elle-même se perd dans la diversité.

\section{LA DOCTRINE}

Très tôt, les médecins qui utilisaient les rayonnements ionisants à des fins diagnostiques ou thérapeutiques sentirent le besoin dans ce domaine d'une politique de la protection de l'homme et c'est en 1928 que fut créée, sous les auspices du Congrès international de radiologie, la Commission internationale de protection radiologique (CIPR).

Depuis cette époque cette commission, tout en continuant à se préoccuper des problèmes posés par la radiologie médicale, a vu peu à peu s'étendre son domaine d'attribution aux autres utilisations des rayonnements 
au fur et à mesure que se multiplièrent les sources et que se développèrent les applications de l'énergie nucléaire. C'est en 1950 qu'elle se réorganisa de façon à couvrir plus efficacement l'ensemble de ce domaine dans lequel elle a toujours été considérée comme l'organisme compétent pour définir et pour recommander la conduite à tenir en vue de protéger les individus, leurs descendants et le genre humain dans son ensemble.

La CIPR a publié régulièrement de 1928 à 1980 des Recommandations dont on peut suivre l'évolution en fonction de celle de nos connaissances sur les effets pathologiques des rayonnements. Cependant, dès 1955 [5], les premiers concepts et les grandes lignes d'une philosophie de la protection sont arrêtés. En 1966 la publication n० 9 [6] fait connaître un premier approfondissement de sa doctrine. En 1977 [7], après avoir analysé les très importantes informations acquises au cours de la décade 1965-1976, elle publie une révision de ses principes fondamentaux qui marque une date importante dans l'élaboration des bases d'une politique rationnelle et cohérente de la protection contre les rayonnements ionisants.

Il est difficile de résumer en quelques lignes cette doctrine et les bases sur lesquelles elle s'appuie. Cependant, en ne retenant que l'essentiel, on peut souligner trois idées :

1. Dans un effort remarquable de généralisation fondée sur des données expérimentales et théoriques, la Commission a défini, en coopération avec la Commission internationale des unités et mesures radiologiques, des concepts simples, en nombre limité, se traduisant par des grandeurs susceptibles d'être évaluées directement ou indirectement par des mesures physiques et correspondant à une expression quantitative des risques pour la santé de l'homme (dose absorbée, équivalent de dose, dose engagée, dose effective, dose collective) ;

2. L'étude des effets pathologiques des rayonnements permet de les classer en deux catégories selon les lois qui régissent leur apparition:

- ceux pour lesquels la relation causale entre dose et effet est de nature déterministe. Ces effets n'apparaissent jamais au-dessous d'un certain seuil de dose,

- ceux pour lesquels la relation causale entre dose et effet est de nature probabiliste (induction d'affections malignes chez l'enfant et l'adulte et effets génétiques). La probabilité d'apparition de l'effet est fonction de la dose. Comme ces affections ou anomalies existent déjà à l'état naturel avec une fréquence non négligeable, il devient impossible au-dessous d'un certain niveau de dose de mettre en évidence l'action des rayonnements. Dans cette zone d'incertitude au niveau des faibles doses, il est pourtant possible d'affirmer qu'une relation linéaire et sans seuil présentant une pente judicieusement choisie en fonction des observations faites à moyennes et fortes doses permet de faire une évaluation de la "limite supérieure" du risque ;

3. Compte tenu de ce que l'on a ainsi appris sur la nature et sur les lois d'apparition des effets pathologiques des rayonnements, plus particulièrement de ceux de la deuxième catégorie (effets dits "aléatoires"), la CIPR a défini les trois principes fondamentaux sur lesquels doit reposer la politique de protection de l'homme :

- toute activité (médicale, scientifique ou industrielle) susceptible d'entraîner une exposition de l'homme aux rayonnements doit être justifiée ; 
- si elle est justifiée, cette activité doit s'exercer dans des conditions de protection telles que les expositions soient maintenues au niveau le plus bas que l'on puisse raisonnablement atteindre compte tenu des facteurs économiques et sociaux ;

- les doses reçues par les individus ne devraient pas dépasser les limites recommandées par la Commission. Ces limites ont été déterminées de façon à satisfaire simultanément à deux conditions :

- être inférieures pour tout tissu de l'organisme (dose à l'organe) aux seuils d'apparition des effets "déterministes",

- être suffisamment faibles au niveau de l'ensemble des différents tissus (dose effective) pour que la limite supérieure du risque éventuel total d'apparition des effets "probabilistes" n'entraîne pas de dommage supérieur à celui qui est couramment accepté soit par les travailleurs dans les professions dites sûres, soit par le public dans sa vie de tous les jours.

Ces limites de dose individuelle, faciles à appliquer avec une marge de sécurité dans le cas de l'exposition externe aux rayonnements, se traduisent dans le cas de l'exposition interne par des "limites annuelles d'incorporation" pour les différents radio-éléments susceptibles d'être inhalés ou ingérés.

Cette doctrine de la CIPR a toujours reçu l'adhésion des plus éminents experts ; tous les pays l'ont choisie comme base de leur réglementation de protection contre les rayonnements ionisants. Depuis vingt-cinq ans que l'essentiel de cette doctrine est appliquée dans tous les pays où l'énergie nucléaire s'est développée, elle a largement fait la preuve de son efficacité tant dans le domaine de l'hygiène professionnelle que dans celui de l'hygiène publique.

En face d'une doctrine aussi claire et aussi structurée, on ne peut qu'être déçu lorsqu'on aborde la protection contre les nuisances non radiologiques.

Tout d'abord, il n'a existé aucune autorité scientifique internationale ayant le recul et l'expérience nécessaires pour pouvoir recommander une philosophie, une politique générale analogue à celle que propose la CIPR. II existe des réglementations nationales qui se contentent d'imposer des règles générales de prévention surtout en hygiène professionnelle et fixent, dans quelques cas très peu nombreux, des valeurs limites de concentration des substances toxiques dans l'air à respecter obligatoirement. En France, par exemple, ces limites obligatoires n'ont été imposées que pour quatre produits: l'amiante, le benzène, le chlorure de vinyle et l'oxyde de carbone. Pour les autres toxiques, il existe des valeurs limites proposées à titre indicatif, pour l'exposition professionnelle, par l'Institut national de recherche et de sécurité (INRS) qui présente, en les tenant à jour, les listes des valeurs établies aux Etats-Unis et en Union Soviétique [8].

Les valeurs américaines adoptées par la Conférence des hygiénistes industriels gouvernementaux doivent être interprétées comme suit :

- il s'agit de valeurs expérimentales déduites tant de la pratique industrielle que de l'expérimentation animale et autant que possible des deux;

- elles ne sont valables que pour un seul contaminant à la fois ; 
- les critères de détermination ne sont pas les mêmes d'une substance à l'autre et ne possèdent pas, de ce fait, de "dénominateur commun". Pour certaines substances, le seuil sera établi sur des considérations subjectives telles que le confort ou l'inconfort de l'ambiance de travail. Pour d'autres sur l'observation caractérisée de troubles à court terme ou à long terme ;

- il en résulte que ces valeurs n'ayant pas toutes la même signification ne peuvent déterminer une soi-disant frontière entre les ambiances dangereuses et celles qui ne le sont pas;

- moins encore, elles ne prétendent pas constituer une échelle de toxicités relatives comparées ;

- ce tableau définit, en revanche, les conditions sous lesquelles on peut estimer que la presque totalité des ouvriers peut être exposée, jour après jour, sans effet défavorable, les valeurs limites étant des valeurs moyennes pondérées pour une durée de travail de 7 à 8 heures par jour et de 40 heures par semaine.

Si la concentration moyenne pondérée en fonction du temps est la méthode la plus satisfaisante et la plus pratique pour maintenir au-dessous des concentrations limites les polluants présents dans l'air, elle ne convient cependant pas dans tous les cas. Pour des substances à action particulièrement rapide, il est plus approprié de fixer la valeur limite en tenant compte de la rapidité de la réaction. II convient alors de déterminer une valeur dite "plafond" qui ne doit être dépassée en aucun cas et permet de mieux garantir des concentrations sans danger.

En Union Soviétique, les valeurs limites de concentration sont élaborées à partir des travaux d'organismes scientifiques et validées par le ministère de la Santé. Elles sont publiées dans une norme GOST 12.1.005.76, entrée en vigueur le $1^{\text {er }}$ janvier 1977 et complétée depuis par trois additifs du ministère de la Santé.

Les valeurs limites de concentration fixées par la norme sont des valeurs "plafonds" qui ne doivent pas être dépassées. La seule exception est l'oxyde de carbone ... Elles s'appliquent à l'atmosphère de la zone de travail de tous les établissements quelle que soit leur nature : locaux industriels, galeries et tranchées, espaces découverts, moyens de transport, etc.

Si on s'applique à comparer pour les mille substances environ qui figurent dans le document de l'INRS les valeurs préconisées aux USA et en URSS, on constate bien des incohérences. Tout d'abord, si on considère les substances toxiques pour lesquelles des valeurs plafonds unt été fixées aux USA, qui, a priori devraient être les plus comparables aux valeurs fixées en URSS, toutes définies comme valeurs plafonds, on a la surprise de constater que sur 41 substances, 9 seulement figurent dans la liste des substances toxiques retenues en URSS. Pour ces 9 substances les valeurs sont pratiquement les mêmes pour 4 d'entre elles, les autres diffèrent d'un facteur allant de 6 à 48 . Le phénomène est général ; pour les autres toxiques de la liste on trouve fréquemment des différences qui s'expriment par des facteurs de 20 à 50 . Ces divergences peuvent s'expliquer soit par des différences dans la conception même de la notion de valeur limite de concentration, soit par des différences de méthodologie et, particulièrement, par l'application généralisée en Union Soviétique des méthodes d'exploration du système nerveux central. 
Les "concentrations maximales admissibles" (dénomination des valeurs limites en URSS) ne semblent pas avoir tout à fait la même signification que les "threshold limit values" des USA. Ces dernières sont définies comme des niveaux auxquels la "presque totalité" des travailleurs peut être exposée sans effet défavorable pour la santé, admettant ainsi qu'il peut exister des sensibilités individuelles susceptibles d'entraîner pour certains de tels effets. Les limites fixées en Union Soviétique sont présentées comme des niveaux auxquels les substances toxiques ne peuvent être la cause, pour aucun travailleur, d'aucun effet défavorable détectable avec les moyens modernes d'exploration clinique [9].

Sur les différences concernant les méthodes et les critères techniques, il ne semble pas qu'un rapprochement important se soit fait jour entre les conceptions américaine et soviétique depuis la parution des travaux de A.A. Letavet dont certains points essentiels avaient été repris par $R$. Truhaut en 1963 [10]. De nombreux spécialistes pensent que les méthodes utilisées par les experts soviétiques pour établir les limites sont trop sensibles et mettent en évidence des réactions physiologiques sans incidence pathologique.

II faut signaler aussi que pour les substances reconnues comme cancérigènes ou soupçonnées d'avoir un tel effet, les lacunes sont considérables surtout dans la liste URSS. Sur 39 substances présentées dans le document de l'INRS comme effectivement ou potentiellement cancérigènes, 17 sont données sans valeur de limite dans la liste USA et 35 dans la liste URSS.

On constate donc que dans ce domaine de la protection contre les substances toxiques en hygiène industrielle, l'unanimité internationale est encore loin d'être réalisée. II faut, cependant, rendre hommage aux efforts de l'Organisation internationale du travail (OIT) et de l'Organisation mondiale de la santé (OMS) qui, après avoir consulté des experts de différents pays, ont publié dès 1969 , pour 24 produits, des "zones de concentrations non dangereuses recommandées pour adoption sur le plan international" [1].

En hygiène publique, la pollution atmosphérique est depuis plusieurs années un sujet d'actualité. La concentration des populations dans les grandes villes et les rejets de substances toxiques de toute sorte par les foyers domestiques, par les différentes sources d'énergie et les procédés industriels, par les moyens de transport, par l'incinération et le traitement des déchets, etc. ont sensibilisé l'opinion à ces problèmes. Les congrès internationaux et nationaux sur ce sujet se sont multipliés et les pouvoirs publics des principales nations ont commencé à élaborer des réglementations nationales qui, pour l'instant, semblent se limiter à des prescriptions générales et à une limitation de l'émission des polluants à la source.

L'élaboration d'une politique générale de limitation des doses au niveau de l'homme semble repoussée à plus tard, le niveau des connaissances en matière de toxicologie chronique aux très petites doses étant encore insuffisant. Cependant, certains pays semblent avoir essayé de définir des limites par analogie avec celles qu'ils avaient adoptées en hygiène industrielle, mais ces essais ont eu des fortunes diverses et la France jusqu'à présent n'a pas suivi ce chemin [11]. Dans cette ligne, cependant, R. Truhaut avait, dès 1963 [10], rappelé les différences les plus importantes 
entre exposition professionnelle et exposition du public dont devrait tenir compte tout essai de dérivation de limites en hygiène publique à partir des valeurs proposées pour les travailleurs:

- le fait que l'exposition de la population est une exposition relativement continue alors que celle des travailleurs par sa discontinuité offre des possibilités de récupération pour l'action de certains agents nocifs ;

- le fait que les travailleurs sont des adultes soumis à des visites d'embauche et à un contrôle médical périodique alors que la population générale comprend des enfants, des vieillards, des malades et des sujets porteurs d'affections non apparentes qui les rendent plus sensibles.

II résulte de ces différences que les "limites de concentration tolérables" dans l'air des villes ou au voisinage des installations industrielles devraient toujours être nettement plus basses que celles concernant spécifiquement les ambiances professionnelles. Mais il faut se rendre à l'évidence, l'état des connaissances et la situation générale ne permettent pas de fixer de telles limites.

$\mathrm{Si}$, donc, on essaye de faire le point, on se rend compte qu'en hygiène publique on est encore moins avancé qu'en hygiène professionnelle et que, dans l'un et l'autre domaine, on est très loin de l'unité et de la cohérence qui caractérisent la politique générale, universellement acceptée, de la protection contre les rayonnements ionisants et les substances radioactives.

II faut signaler, cependant, qu'en 1977, un pas extrêmement important a été franchi au niveau international par la création de la Commission internationale de protection contre les substances mutagènes et carcinogènes dans l'environnement. Fondée sous les auspices de l'Institut de la vie et affiliée à l'Association internationale des sociétés dont l'objectif est l'étude des mutagènes, cette commission se propose d'évaluer les risques pour Phomme des agents chimiques mutagènes et carcinogènes et de recommander une ligne de conduite susceptible de prévenir ou de minimiser les effets nocifs que peut avoir leur présence dans l'environnement. II faut espérer que cet organisme parviendra le plus rapidement possible à ses fins et que la valeur de ses travaux entraînera le consensus international préalable à l'unification des dispositions réglementaires dans les différents pays.

Pour terminer cette étude, il n'est pas inutile de revenir sur deux idées qui ont souvent été développées au cours de ces dernières années et particulièrement en France.

La première est que si l'on compare les possibilités techniques de surveillance du milieu et de l'organisme lui-même à l'égard des deux types de nuisances radiologique et non radiologique, la sensibilité des moyens de détection et de mesure des rayonnements et des substances radioactives est, en général, bien meilleure que celle des techniques utilisées couramment pour détecter et mesurer la pollution non radiologique. Bien que certaines méthodes de laboratoire permettent pour certains toxiques de 
mettre en évidence la présence de quantités très faibles de substance (benzopyrène) dans une seule bouffée de fumée de cigarette par exemple), on a pu établir que le rapport moyen (moyenne géométrique) de la plus faible quantité mesurable en routine au niveau limite à respecter, était de P'ordre de $2.10^{-4}$ pour les substances radioactives et de $7.10^{-2}$ pour les autres nuisances [12]. Ceci assure une marge de sécurité considérable pour le contrôle radiologique des conditions de travail et pour la surveillance radiologique de l'environnement par rapport aux autres nuisances.

La seconde idée, qui pourra servir de conclusion, est que des progrès très importants devraient se manifester dans les années qui viennent pour la détermination de valeurs limites qui pourraient rallier les suffrages de tous lorsqu'il s'agit de ce qu'il est convenu d'appeler les agents génotoxiques, c'est-à-dire susceptibles d'avoir une intéraction avec le génome cellulaire, ce qui peut se traduire par des effets génétiques et cancérigènes. Se fondant sur la similitude qui existe entre les effets génotoxiques des rayonnements ionisants et ceux de ces polluants chimiques, des chercheurs [13] ont conçu une comparaison qui, sous certaines conditions, permettrait d'envisager l'extrapolation des règles admises pour les rayonnements à des règles valables pour ces substances. Certes, bien des incertitudes demeurent mais déjà des équivalences ont été établies pour quelques toxiques et la voie ouverte paraît pleine de promesses.

\section{RÉFÉRENCES BIBLIOGRAPHIQUES}

[1] OMS. Niveaux admissibles d'exposition professionnelle aux substances toxiques

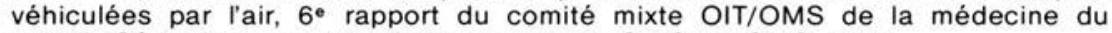
travail. (Série de rapports techniques n॰415). Genève : OMS, 1969.

[2] UNSCEAR. Reports to the General Assembly of the United Nations, Official Records:

13th Session, Suppl. No 17 (A/3838), 1958.

17 th Session, Suppl. No 16 (A/5216), 1962.

19 th Session, Suppl. No 14 (A/5814), 1964.

21 st Session, Suppl. No 14 (A/6314 and corr. 1), 1966.

24 th Session, Suppl. No 13 (A/7613 and corr. 1), 1969.

27 th Session, Suppl. No 25 (A/8725 and corr. 1), 1972.

32nd Session, Suppl. No 20 (A/3240), 1977.

Genève: Nations Unies.

[3] LATARJET R. Le principe de rad-équivalence : condition de validité. In :SFRP. Comparaison quantitative des nuisances mutagènes : rad-équivalence, Paris, 29 mai 1979, Fontenay-aux-Roses: SFRP, 1979.

[4] IARC. Carcinogenic risks, strategies for intervention. (IARC Scientific publications $\left.n^{\circ} 25\right)$. Genève : OMS, 1979.

[5] CIPR. Recommandations (revues le 1er décembre 1954). J. Radiol. Electrol., $1955,36, n^{\circ} 10$ bis.

[6] CIPR. Recommandations. Publication n॰ 9 (1965). Fontenay-aux-Roses : Département de Protection, s.d.

[7] CIPR. Recommandations. Publication n॰ 26 (1977) (version française), Oxford : Pergamon Press, 1979.

[8] INRS. Valeurs limites de concentration des substances toxiques dans l'air. Cahiers de notes documentaires, 1980 , n॰ 98, note 1231 - 98-80. 
[9] ROSCHIN A.V. and TIMOFEEVSKAYA L.A. Chemical substances in the work environment : some comparative aspects of USSR and US hygienic standards. Ambio, 1975, 4, 30-33.

[10] TRUHAUT R. Les problèmes des limites tolérables pour les substances toxiques dans l'industrie. Divergences et points d'accord à l'échelle internationale. In : $14^{\mathrm{e}}$ Congrès international de médecine du travail (Excerpta Medica Foundation, International Congress series n॰62) - Amsterdam : Excerpta Medica, 1963.

[11] ROUSSEL A. La pollution atmosphérique. Encycl. Med. Chir., Paris, Intoxications, 1978, 1, 16001.

[12] PELLERIN P. et MORONI J.P. Down to what threshold is it reasonable to measure the nuisance in the interest of public health? Colloque de Berlin, Oct. 1979 .

[13] LATARJET R. Vers une évaluation quantitative des risques génotoxiques associés aux divers modes de production d'énergie. In : SFEN - Colloque sur les risques sanitaires des différentes énergies, Paris, janvier 1980. Saint-Etienne : GEDIM, 1980, 39-51. 